K. KAWAMURA

KODAI MATH. J.

8 (1985), $70-78$

\title{
A NOTE ON THE RECURRENT RELATIONS FOR THE BIVARIATE POISSON DISTRIBUTION
}

\author{
By KaZUTOMO KAWAMURA
}

\section{Introduction.}

It is a well known fact that the Poisson density: $p(k ; \lambda)=\left(\lambda^{k} / k !\right) e^{-\lambda}(k=$ $0,1, \cdots)$, satisfies the relation $k p(k ; \lambda)=\lambda p(k-1 ; \lambda)(k \geqq 1)$. And also, we know recurrence relations for the bivariate Poisson density. The relations have been expressed as in $\operatorname{Kot} z$ [3]

$$
\begin{aligned}
& k p(k, l)=\lambda_{10} p(k-1, l)+\lambda_{11} p(k-1, l-1), \\
& l p(k, l)=\lambda_{01} p(k, l-1)+\lambda_{11} p(k-1, l-1)
\end{aligned}
$$

where $p(k, l)(k, l=0,1,2, \cdots)$ is a density of the bivariate Poisson distribution, and $\lambda_{10}, \lambda_{01}, \lambda_{11}$ are its nonnegative three parameters.

In this paper, we will refine the recurrence relations and investigate the role of the relations. It will be shown that the bivariate Poisson density satisfies the recurrence relations and conversely, if we assume the relations, we will get the density $p(k, l)$. But it will be recognized that the recurrence relations (for the bivariate Poisson distribution) are self duplicated and they may be rearranged as minimum conditions as to get the density.

\section{Notations and definitions.}

$p(k ; \lambda)(k=0,1,2, \cdots)$ : a density of the univariate Poisson distribution, where $\lambda$ is its nonnegative parameter.

$p(k, l)(k, l=0,1,2, \cdots)$ : a density of the bivariate Poisson distribution.

$\lambda_{10}, \lambda_{01}, \lambda_{11}$ : three parameters for the bivariate Poisson distribution.

The bivariate density for $k, l=0,1,2, \cdots$, will be represented by

$$
\begin{aligned}
& p(k, l)=\sum_{\delta=0}^{k \wedge l} p\left(k-\delta ; \lambda_{10}\right) p\left(l-\delta ; \lambda_{01}\right) p\left(\delta ; \lambda_{11}\right) \\
& =\sum_{\delta=0}^{k \wedge l} \frac{\lambda_{10}{ }^{k-\delta} \lambda_{01}{ }^{l-\delta} \lambda_{11}{ }^{\delta}}{(k-\delta) !(l-\delta) ! \delta !} e^{-\lambda_{10}-\lambda_{01}-\lambda_{11}},
\end{aligned}
$$

see, Kawamura [2].

Received March 2, 1984 
Lemma 1. The distribution of bivariate Poisson $p(k, l)$ satisfies the recurrence relations,

$$
\begin{aligned}
& k p(k, l)=\lambda_{10} p(k-1, l)+\lambda_{11} p(k-1, l-1), \\
& l p(k, l)=\lambda_{01} p(k, l-1)+\lambda_{11} p(k-1, l-1)
\end{aligned}
$$

for every integers $k, l=1,2, \cdots$.

Proof. This lemma describes the bivariate density $p(k, l)(k, l=1,2, \cdots)$ has the property that the density of a point $(k, l)$ is calculated by the two densities of the neighbouring points $((k-1, l)$ or $(k, l-1))$ and $(k-1, l-1)$ for every $k, l=1,2, \cdots$.

In fact, the density $p(k, l)$ has been expressed as

$$
p(k, l)=\sum_{\delta=0}^{k \wedge l} p\left(k-\delta ; \lambda_{10}\right) p\left(l-\delta ; \lambda_{01}\right) p\left(\delta ; \lambda_{11}\right) .
$$

If we assume $k \leqq l$, then we have

$$
\begin{aligned}
& p(k, l)=\sum_{\delta=0}^{k} p\left(k-\delta ; \lambda_{10}\right) p\left(l-\delta ; \lambda_{01}\right) p\left(\delta ; \lambda_{11}\right) \\
& =p\left(k ; \lambda_{10}\right) p\left(l ; \lambda_{01}\right) p\left(0 ; \lambda_{11}\right) \\
& \quad+\cdots \\
& \quad+p\left(0 ; \lambda_{10}\right) p\left(l-k ; \lambda_{01}\right) p\left(k ; \lambda_{11}\right), \\
& k p(k, l)=k p\left(k ; \lambda_{10}\right) p\left(l ; \lambda_{01}\right) p\left(0 ; \lambda_{11}\right) \\
& \quad+(k-1) p\left(k-1 ; \lambda_{10}\right) p\left(l-1 ; \lambda_{01}\right) p\left(1 ; \lambda_{11}\right) \\
& +(k-2) p\left(k-2 ; \lambda_{10}\right) p\left(l-2 ; \lambda_{01}\right) p\left(2 ; \lambda_{11}\right) \\
& +\cdots \\
& +1 p\left(1 ; \lambda_{10}\right) p\left(l-k+1 ; \lambda_{01}\right) p\left(k-1 ; \lambda_{11}\right) \\
& \quad+1 p\left(k-1 ; \lambda_{10}\right) p\left(l-1 ; \lambda_{01}\right) p\left(1 ; \lambda_{11}\right) \\
& \quad+2 p\left(k-2 ; \lambda_{10}\right) p\left(l-2 ; \lambda_{01}\right) p\left(2 ; \lambda_{11}\right) \\
& +\cdots \\
& +(k-1) p\left(1 ; \lambda_{10}\right) p\left(l-k+1 ; \lambda_{01}\right) p\left(k-1 ; \lambda_{11}\right) \\
& +k p\left(0 ; \lambda_{10}\right) p\left(l-k ; \lambda_{01}\right) p\left(k ; \lambda_{11}\right) \\
& \quad=\lambda_{10} p\left(k-1 ; \lambda_{10}\right) p\left(l ; \lambda_{01}\right) p\left(0 ; \lambda_{11}\right) \\
& +\lambda_{10} p\left(k-2 ; \lambda_{10}\right) p\left(l-1 ; \lambda_{01}\right) p\left(1 ; \lambda_{11}\right) \\
& +\cdots
\end{aligned}
$$




$$
\begin{aligned}
& +\lambda_{10} p\left(0 ; \lambda_{10}\right) p\left(l-k ; \lambda_{01}\right) p\left(k-1 ; \lambda_{11}\right) \\
& \quad+\lambda_{11} p\left(k-1 ; \lambda_{10}\right) p\left(l-1 ; \lambda_{01}\right) p\left(0 ; \lambda_{11}\right) \\
& \quad+\lambda_{11} p\left(k-2 ; \lambda_{10}\right) p\left(l-2 ; \lambda_{01}\right) p\left(1 ; \lambda_{11}\right) \\
& +\cdots \\
& +\lambda_{11} p\left(0 ; \lambda_{10}\right) p\left(l-k ; \lambda_{01}\right) p\left(k-1 ; \lambda_{11}\right) \\
& \quad=\lambda_{10}{ }^{k-1 \wedge l} p\left(k-1-\delta ; \lambda_{10}\right) p\left(l-\delta ; \lambda_{01}\right) p\left(\delta ; \lambda_{11}\right) \\
& \quad+\lambda_{11}{ }^{k-1 \wedge l-1} \sum_{\delta=0}^{l-1} p\left(k-1-\delta ; \lambda_{10}\right) p\left(l-1-\delta ; \lambda_{01}\right) p\left(\delta ; \lambda_{11}\right) \\
& =\lambda_{10} p(k-1, l)+\lambda_{11} p(k-1, l-1) .
\end{aligned}
$$

And similarly, if we assume $k>l$, then by the definition of the density:

$$
\begin{aligned}
p(k, l)= & \sum_{\delta=0}^{l} p\left(k-\delta ; \lambda_{10}\right) p\left(l-\delta ; \lambda_{01}\right) p\left(\delta ; \lambda_{11}\right) \\
= & p\left(k ; \lambda_{10}\right) p\left(l ; \lambda_{01}\right) p\left(0 ; \lambda_{11}\right) \\
& +\cdots \\
& +p\left(k-l ; \lambda_{10}\right) p\left(0 ; \lambda_{01}\right) p\left(l ; \lambda_{11}\right),
\end{aligned}
$$

we have

$$
\begin{aligned}
& k p(k, l)=k p\left(k ; \lambda_{10}\right) p\left(l ; \lambda_{01}\right) p\left(0 ; \lambda_{11}\right) \\
& \quad+(k-1) p\left(k-1 ; \lambda_{10}\right) p\left(l-1 ; \lambda_{01}\right) p\left(1 ; \lambda_{11}\right) \\
& +(k-2) p\left(k-2 ; \lambda_{10}\right) p\left(l-2 ; \lambda_{01}\right) p\left(2 ; \lambda_{11}\right) \\
& +\cdots \\
& +(k-l) p\left(k-l ; \lambda_{10}\right) p\left(0 ; \lambda_{01}\right) p\left(l ; \lambda_{11}\right) \\
& \quad+1 p\left(k-1 ; \lambda_{10}\right) p\left(l-1 ; \lambda_{01}\right) p\left(1 ; \lambda_{11}\right) \\
& \quad+2 p\left(k-2 ; \lambda_{10}\right) p\left(l-2 ; \lambda_{01}\right) p\left(2 ; \lambda_{11}\right) \\
& \quad+\cdots \\
& +l p\left(k-l ; \lambda_{10}\right) p\left(0 ; \lambda_{01}\right) p\left(l ; \lambda_{11}\right) \\
& \quad=\lambda_{10} p\left(k-1 ; \lambda_{10}\right) p\left(l ; \lambda_{01}\right) p\left(0 ; \lambda_{11}\right) \\
& \quad+\lambda_{10} p\left(k-2 ; \lambda_{10}\right) p\left(l-1 ; \lambda_{01}\right) p\left(1 ; \lambda_{11}\right) \\
& \quad+\cdots \\
& +\lambda_{10} p\left(k-1-l ; \lambda_{10}\right) p\left(0 ; \lambda_{01}\right) p\left(l ; \lambda_{11}\right)
\end{aligned}
$$




$$
\begin{aligned}
& \quad+\lambda_{11} p\left(k-1 ; \lambda_{10}\right) p\left(l-1 ; \lambda_{01}\right) p\left(0 ; \lambda_{11}\right) \\
& +\lambda_{11} p\left(k-2 ; \lambda_{10}\right) p\left(l-2 ; \lambda_{01}\right) p\left(1 ; \lambda_{11}\right) \\
& +\cdots \\
& +\lambda_{11} p\left(k-l ; \lambda_{10}\right) p\left(0 ; \lambda_{01}\right) p\left(l-1 ; \lambda_{11}\right) \\
& =\lambda_{10} \sum_{\delta=0}^{k-1 \wedge l} p\left(k-1-\delta ; \lambda_{10}\right) p\left(l-\delta ; \lambda_{01}\right) p\left(\delta ; \lambda_{11}\right) \\
& \quad+\lambda_{11} \sum_{\delta=0}^{k-1 \wedge l-1} p\left(k-1-\delta ; \lambda_{10}\right) p\left(l-1-\delta ; \lambda_{01}\right) p\left(\delta ; \lambda_{11}\right) \\
& =\lambda_{10} p(k-1, l)+\lambda_{11} p(k-1, l-1) .
\end{aligned}
$$

Therefore, we have finished the proof of the relation (1). Let us proceed to finish the proof of the lemma. But we can easily obtain the other relation:

$$
l p(k, l)=\lambda_{10} p(k-1, l)+\lambda_{11} p(k-1, l-1)
$$

for every $k, l=0,1,2, \cdots$, by a similar consideration just proved. Then, the proof of our lemma may be finished.

\section{Main results.}

LEMMA 2. The density of bivariate Poisson distribution $p(k, l)$ satisfies the recurrence relations:

$$
\begin{array}{ll}
k p(k, 0)=\lambda_{10} p(k-1,0) & (k=1,2, \cdots), \\
l p(0, l)=\lambda_{01} p(0, l-1) & (l=1,2, \cdots)
\end{array}
$$

and for the original point $(k=0, l=0)$ consısts with a density

$$
p(0,0)=e^{-\lambda_{10}-\lambda_{01}-\lambda_{11}} \text {. }
$$

Proof. If we assume $k \geqq 1$, then we have

$$
\begin{aligned}
k p(k, 0) & =k p\left(k ; \lambda_{10}\right) p\left(0 ; \lambda_{01}\right) p\left(0 ; \lambda_{11}\right) \\
& =\lambda_{10} p\left(k-1 ; \lambda_{10}\right) p\left(0 ; \lambda_{01}\right) p\left(0 ; \lambda_{11}\right) \\
& =\lambda_{10} p(k-1,0) .
\end{aligned}
$$

And, in the same way, if we assume $l \geqq 1$, then we have

$$
l p(0, l)=\lambda_{01} p(0, l-1) .
$$

For the original point $(k=0, l=0)$, the value of the bivariate Poisson density $p(0,0)$ is given directly by the definition. 


$$
\begin{aligned}
p(0,0) & =p\left(0 ; \lambda_{10}\right) p\left(0 ; \lambda_{01}\right) p\left(0 ; \lambda_{11}\right) \\
& =e^{-\lambda_{10}-\lambda_{01}-\lambda_{11}} .
\end{aligned}
$$

Summarize the lemma 1 and lemma 2, we can express the next theorem.

THEOREM 1. If $p(k, l)(k, l=0,1,2, \cdots)$ is assumed to be a density of $a$ bivariate Poisson distribution with parameters $\lambda_{10}, \lambda_{01}$ and $\lambda_{11}$ then the density function satisfies the relations; (1), (2), (3), (4) and (5).

In the following lines, we would like to discuss whether the converse assertion of the theorem is satisfied or not. Because, if we wish to calculate the function $p(k, l)$ for all probability space $(k, l=0,1,2, \cdots)$, we wonder how many relations for the function are needed. The answer will be given in the following theorem.

THEOREM 2. If a function $p(k, l)$ satisfies the next relations;

$$
\begin{aligned}
& k p(k, l)=\lambda_{10} p(k-1, l)+\lambda_{11} p(k-1, l-1) \quad \text { for } \quad k, l=1,2, \cdots, \\
& k p(k, 0)=\lambda_{10} p(k-1,0) \quad \text { for } \quad k=1,2, \cdots, \\
& l p(0, l)=\lambda_{01} p(0, l-1) \quad \text { for } \quad l=1,2, \cdots,
\end{aligned}
$$

and

$$
p(0,0)=e^{-\lambda_{10}-\lambda_{01}-\lambda_{11}}
$$

then the function consists with the density of bivariate Poisson distribution

$$
p(k, l)=\sum_{\delta=0}^{k \wedge 1} p\left(k-\delta ; \lambda_{10}\right) p\left(l-\delta ; \lambda_{01}\right) p\left(\delta ; \lambda_{11}\right)
$$

for every $k, l=0,1,2, \cdots$.

Proof. From the relation $(3) ; k p(k, 0)=\lambda_{10} p(k-1,0)(k \geqq 1)$ and $(5) ; p(0,0$; $=e^{-\lambda_{10}-\lambda_{01}-\lambda_{11}}$, we have

$$
\begin{aligned}
p(k, 0) & =\frac{\lambda_{10}{ }^{k}}{k !} e^{-\lambda_{10}-\lambda_{01}-\lambda_{11}} \\
& =p\left(k ; \lambda_{10}\right) p\left(0 ; \lambda_{01}\right) p\left(0 ; \lambda_{11}\right) \quad \text { for all } k \geqq 0,
\end{aligned}
$$

the right side of the equality is consistent with the density of bivariate Poisson distribution $P(\lambda)$.

From the relation $(4) ; l p(0, l)=\lambda_{01} p(0, l-1)(l \geqq 1)$ and (5), we have

$$
\begin{aligned}
p(0, l) & =l \frac{\lambda_{01}{ }^{l}}{l !} e^{\lambda_{10}-\lambda_{01}-\lambda_{11}} \\
& =p\left(0 ; \lambda_{10}\right) p\left(l ; \lambda_{01}\right) p\left(0 ; \lambda_{11}\right) \quad \text { for all } l \geqq 0,
\end{aligned}
$$


the right side of the equality is consistent with the density of $P(\lambda)$.

We have already derived in (6) the function on the $X$ axis (on the line $Y=0) ; p(k, 0)(k \geqq 0)$ to be consistent with the bivariate density. In the following lines, we will derive the value of the function on the line $Y=l ; p(k, l)$ $(k \geqq 0)$, and the function is consistent with the bivariate density, by the induction for $l \geqq 1$.

Let's prove the function to be consistent with the density on the line $Y=n$, assuming the function to be consistent with the density on the line $Y=n-1$, that is ;

$$
p(k, n-1)=\sum_{\delta=0}^{k \wedge n-1} p\left(k-\delta ; \lambda_{10}\right) p\left(n-1-\delta ; \lambda_{01}\right) p\left(\delta ; \lambda_{11}\right) \quad \text { for all } k \geqq 0 .
$$

We have already shown $p(0, n-1)$ and $p(0, n)$ to be consistent with the density by the equality (7).

We would like to use the second induction for $m \geqq 1$ and prove that the function $p(m, n)$ for $m \geqq 1$ is consistent with the density.

Let's assume the value of the function $p(m-1, n)$ to be consistent with the density:

$$
p(m-1, n)=\sum_{\delta=0}^{m-1 \wedge n} p\left(m-1-\delta ; \lambda_{10}\right) p\left(n-\delta ; \lambda_{01}\right) p\left(\delta ; \lambda_{11}\right) .
$$

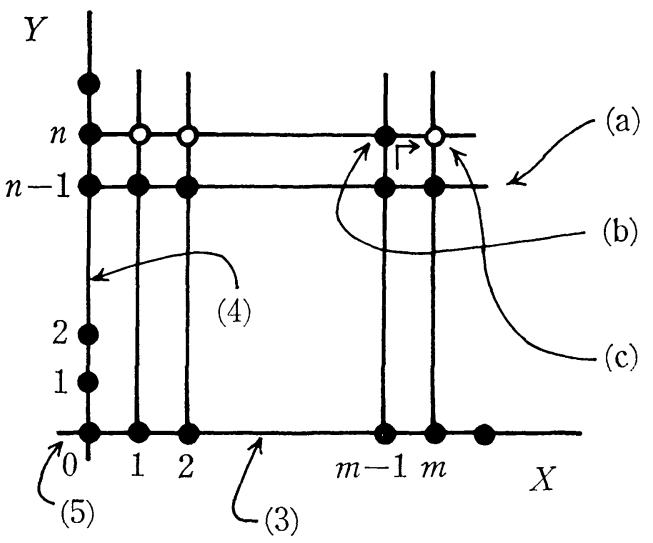

Figure (a) We assume that the function $p(k, n-1)(k \geqq 1)$ on the line $Y=n-1$ is consistent with the density by the assumption (8).

(b) Now we assume the value $p(m-1, n)$ to be consistent with the density by (9).

(c) From the relation (1), we can calculate the value of the function $p(m, n)$ by (8) and (9).

Putting $k=m-1$ in the assumption (8), we have

$$
p(m-1, n-1)=\sum_{\delta=0}^{m-1 \wedge n-1} p\left(m-1-\delta ; \lambda_{10}\right) p\left(n-1-\delta ; \lambda_{01}\right) p\left(\delta ; \lambda_{11}\right) .
$$

From the recurrence relation (1),

$$
m p(m, n)=\lambda_{10} p(m-1, n)+\lambda_{01} p(m-1, n-1)
$$


We can derive that the function $p(m, n)$ is consistent with the density:

$$
p(m, n)=\sum_{\delta=0}^{m \wedge n} p\left(m-\delta ; \lambda_{10}\right) p\left(n-\delta ; \lambda_{01}\right) p\left(\delta ; \lambda_{11}\right),
$$

and we will finish the second induction for $m \geqq 1$.

Let's prove (12) by (11).

$$
\begin{aligned}
m p(m, n)= & \lambda_{10} \sum_{\delta=0}^{m-1 \wedge n} p\left(m-1-\delta ; \lambda_{10}\right) p\left(n-\delta ; \lambda_{01}\right) p\left(\delta ; \lambda_{11}\right) \\
& +\lambda_{11} \sum_{\delta=0}^{m-1 \wedge n-1} p\left(m-1-\delta ; \lambda_{10}\right) p\left(n-1-\delta ; \lambda_{01}\right) p\left(\delta ; \lambda_{11}\right) \\
= & \sum_{\delta=0}^{m-1 \wedge n} \lambda_{10} p\left(m-1-\delta ; \lambda_{10}\right) p\left(n-\delta ; \lambda_{01}\right) p\left(\delta ; \lambda_{11}\right) \\
& +\sum_{\delta=0}^{m-1 \wedge n-1} p\left(m-1-\delta ; \lambda_{10}\right) p\left(n-1-\delta ; \lambda_{01}\right) \lambda_{11} p\left(\delta ; \lambda_{11}\right) \\
= & \sum_{\delta=0}^{m-1 \wedge n}(m-\delta) p\left(m-\delta ; \lambda_{10}\right) p\left(n-\delta ; \lambda_{01}\right) p\left(\delta ; \lambda_{11}\right) \\
& +\sum_{\delta=0}^{m-1 \wedge n-1}(\delta+1) p\left(m-1-\delta ; \lambda_{10}\right) p\left(n-1-\delta ; \lambda_{01}\right) p\left(\delta+1 ; \lambda_{11}\right) \\
= & \sum_{\delta=0}^{m-1 \wedge n}(m-\delta) p\left(m-\delta ; \lambda_{10}\right) p\left(n-\delta ; \lambda_{01}\right) p\left(\delta ; \lambda_{11}\right) \\
& +\sum_{\delta=0}^{(m-1 \wedge n-1)+1} \delta p\left(m-\delta ; \lambda_{10}\right) p\left(n-\delta ; \lambda_{01}\right) p\left(\delta ; \lambda_{11}\right) .
\end{aligned}
$$

If we assume $m \leqq n$, then we have

$$
\begin{aligned}
m p(m, n)= & \sum_{\delta=0}^{m-1}(m-\delta) p\left(m-\delta ; \lambda_{10}\right) p\left(n-\delta ; \lambda_{01}\right) p\left(\delta ; \lambda_{11}\right) \\
& +\sum_{\delta=0}^{m} \delta p\left(m-\delta ; \lambda_{10}\right) p\left(n-\delta ; \lambda_{01}\right) p\left(\delta ; \lambda_{11}\right) \\
= & m \sum_{\delta=0}^{m} p\left(m-\delta ; \lambda_{10}\right) p\left(n-\delta ; \lambda_{01}\right) p\left(\delta ; \lambda_{11}\right) .
\end{aligned}
$$

And, we have

$$
p(m, n)=\sum_{\delta=0}^{m \wedge n} p\left(m-\delta ; \lambda_{10}\right) p\left(n-\delta ; \lambda_{01}\right) p\left(\delta ; \lambda_{11}\right) .
$$

If we assume on the contrary $m>n$, then we have

$$
m p(m, n)=\sum_{\delta=0}^{n}(m-\delta) p\left(m-\delta ; \lambda_{10}\right) p\left(n-\delta ; \lambda_{01}\right) p\left(\delta ; \lambda_{11}\right)
$$




$$
\begin{aligned}
& +\sum_{\delta=0}^{n} \delta p\left(m-\delta ; \lambda_{10}\right) p\left(n-\delta ; \lambda_{01}\right) p\left(\delta ; \lambda_{11}\right) \\
= & m \sum_{\delta=0}^{n} p\left(m-\delta ; \lambda_{10}\right) p\left(n-\delta ; \lambda_{01}\right) p\left(\delta ; \lambda_{11}\right) .
\end{aligned}
$$

And also, we have the same result

$$
p(m, n)=\sum_{\delta=0}^{m \wedge n} p\left(m-\delta ; \lambda_{10}\right) p\left(n-\delta ; \lambda_{01}\right) p\left(\delta ; \lambda_{11}\right) \quad(m \geqq 0) .
$$

From the assertion just finished, by the second induction for $m=0,1,2, \cdots$ that is, the function $p(m, n)$ for $m=0,1,2, \cdots$ is consistent with the density for fixed $n$ in the first induction; we will finish the first induction for $n=$ $0,1,2, \cdots$.

COROLlARY 2-1. If we assume the recurrence relations (1), (3), (4) and $\sum_{k, l=0}^{\infty} p(k, l)=1$ hold for a function $p(k, l)$, then we can derive that the function is consistent with the density.

Proof. Put the function's value as $p_{000}$ at the origin $(k=0, l=0)$. Then, by the relations (3) and (4), we can derive all the value on $X$ and $Y$ axes.

By the same argument with the induction for $Y=n$ in the preceding theorem, we may derive

$$
p(m, n)=\sum_{\delta=0}^{m \wedge n} \frac{\lambda_{10}{ }^{m-\delta} \lambda_{01}{ }^{n-\delta} \lambda_{11}{ }^{\delta}}{(m-\delta) !(n-\delta) ! \delta !} \cdot p_{000}
$$

for every $m, n \geqq 0$. Finally, using the property of a bivariate density

$$
\sum_{k, l} p(k, l)=1
$$

we can easily derive $p(0,0)=p_{000}=e^{-\lambda_{10}-\lambda_{01}-\lambda_{11}}$.

The assertion of theorem 2 may hold, replacing the relation (1) by the relation (2), and the assertion of corollary 2-1 also hold. To summarize these results, we can express a theorem and a corollary as follows.

THEOREM 3. If a function $p(k, l)$ satisfies the relations (1) or (2) plus (3), (4) and (5), then we can derive

$$
p(k, l)=\sum_{\delta=0}^{k \wedge l} p\left(k-\delta ; \lambda_{10}\right) p\left(l-\delta ; \lambda_{01}\right) p\left(\delta ; \lambda_{11}\right) \quad \text { for every } \quad k, l \geqq 0 .
$$

COROLLARY 3-1. If we assume the recurrence relations (1) or (2) plus (3), (4) and $\sum_{k, l} p(k, l)=1$ holds on a function $p(k, l)$, then we can derive the function is consistent with the density as in theorem 3. 


\section{Conclusion.}

Finally, if we combine our theorem 1 and theorem 3, then we have a theorem which is the main result of this paper.

THEOREM 4. To derive the density of the bivariate Poisson distribution, the necessary and sufficient conditions are expressed as (1) or (2) plus (3), (4) and (5).

The author has considered the result of this paper would be generalized in the multivariate case. The relations for the density of the multivariate Poisson distribution will be announced later in the journal.

\section{REFERENCES}

[1] Feller, W., An introduction to probability theory and its applications, Vol. 1, sec. ed. 6th print (1961).

[2] Kawamura, K., The structure of bivariate Poisson distribution, Kōdai Math. Sem. Rep., 25 (2) (1973), 246-256.

[3] Johnson, Norman L. AND Kotz Samuel, Discrete distributions, Houghton Mifflin Co. (1969).

Department of Mathematics

TOKyo Institute of TECHNOLOgy 